\title{
Quantum Transport Simulation of the DOS function, Self- Consistent Fields and Mobility in MOS Inversion Layers
}

\author{
DRAGICA VASILESKA ${ }^{\mathrm{a}}$, TERRY ELDRIDGE ${ }^{\mathrm{b}}$, PAOLO BORDONE $^{\mathrm{c}}$ and DAVID K. FERRY ${ }^{\mathrm{a}}$ \\ ${ }^{\mathrm{a} C e n t e r ~ f o r ~ S o l i d-S t a t e ~ E l e c t r o n i c s ~ R e s e a r c h, ~ A r i z o n a ~ S t a t e ~ U n i v e r s i t y ~ T e m p e, ~ A z, ~ 85287-6206, ~ U S A, ~}{ }^{\mathrm{b}}$ McDonnell Douglas Helicopter Sys- \\ tems, 5000 E. McDowell Rd, Mesa, Az, 85215, US, ${ }^{\circ}$ Dipartimento di Fisica ed Instituto Nazionale di Fisica della Materia, Università di \\ Modena, Via Campi 213/A, 41100 Modena, Italy.
}

\begin{abstract}
We describe a simulation of the self-consistent fields and mobility in (100) Si-inversion layers for arbitrary inversion charge densities and temperatures. A nonequilibrium Green's functions formalism is employed for the state broadening and conductivity. The subband structure of the inversion layer electrons is calculated self-consistently by simultaneously solving the Schrödinger, Poisson and Dyson equations. The self-energy contributions from the various scattering mechanisms are calculated within the self-consistent Born approximation. Screening is treated within RPA. Simulation results suggest that the proposed theoretical model gives mobilities which are in excellent agreement with the experimental data.
\end{abstract}

Keywords: Green's functions, mobility, inversion layers, surface-roughness, broadening of the states.

The inversion layer mobility in Si MOSFET is a very important physical quantity that describes the drain current and also serves as a probe to study the electronic transport properties of the quasi-two-dimensional (Q2D) electron gas. Semi-empirical models have been developed to explain the overall mobility behavior at various temperatures and different gate voltages [1,2]. Monte Carlo studies of the two-dimensional electron transport in Si MOS devices have also been performed [3]. However, scaling of the MOS device downwards leads to high surface fields. Then, the degenerate nature of the electrons dominates the transport properties of the structure. The Fermi-Dirac statistics influence the mobility in two ways: through the screening properties of the electron gas and through the distribution function itself. In addition, broadening of the states near the subband thresholds impacts the redistribution of the carriers among vari- ous subbands. To overcome some of the limitations of previous theories, we use a new theoretical model in which the two-dimensional aspects of carrier motion, intersubband transitions, the anisotropy of the acoustic phonon interaction, as well as the broadening of the electronic states and the degenerate nature of the Q2D electron gas are simultaneously taken into account.

The theoretical model is summarized in Fig. 1. The initial potential energy profile is calculated analytically using a variational approach for the electronic quantum limit. The Schrödinger, Poisson and Dyson equations are then iterated for the corresponding unknowns [4], and self-consistency is achieved through the outer iteration. For each iteration, the one-dimensional Schrödinger equation is integrated leftward $\left(\psi_{n}^{<}\right)$and rightward $\left(\psi_{n}^{>}\right)$using a 1D version of the Numerov algorithm [5]. The effective

\footnotetext{
* Work supported by the Office of Naval Research

$\dagger$ Corresponding author. E-mail:vasilesk@imap2.asu.edu
} 


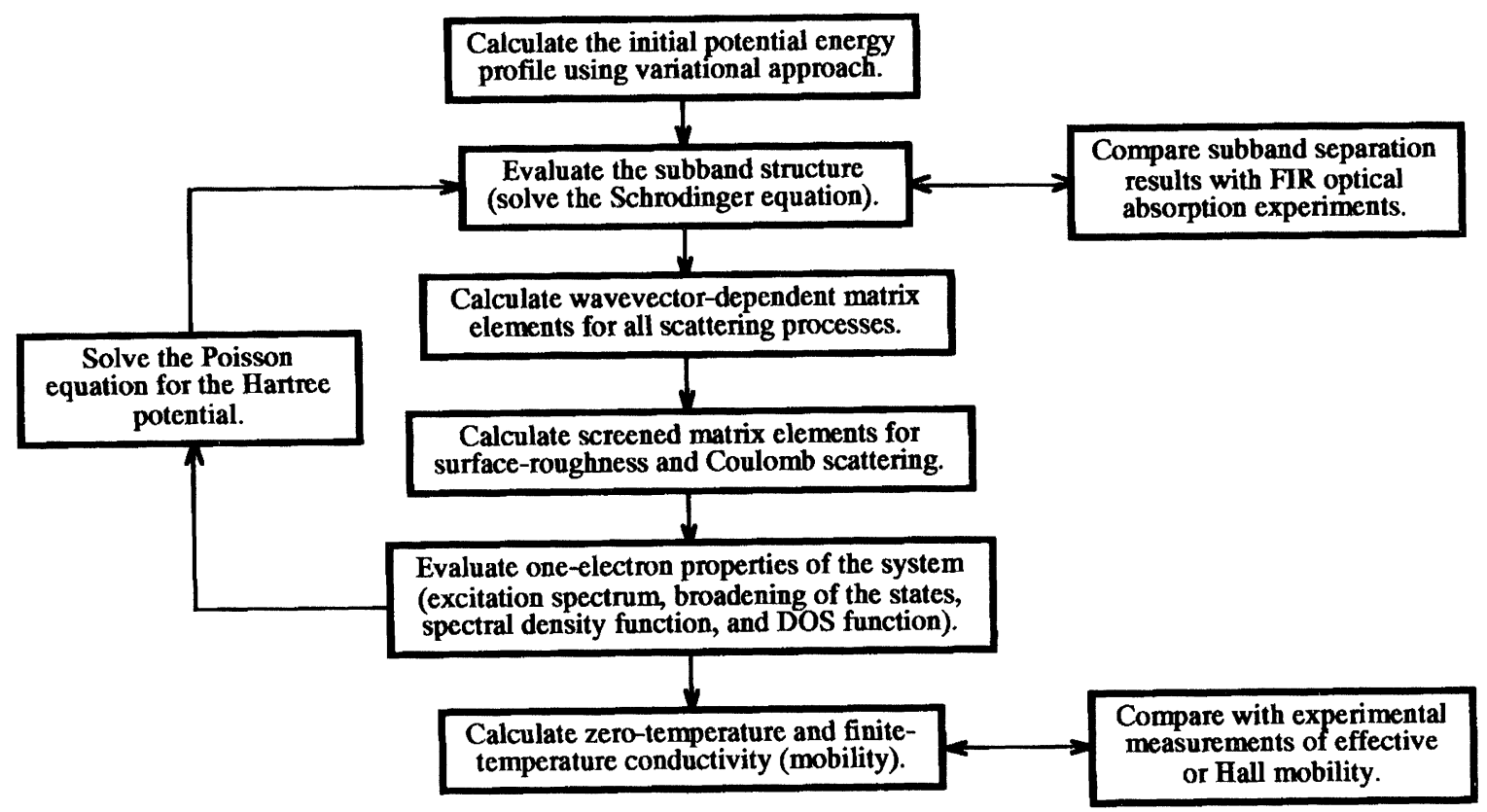

FIGURE 1 Flow-chart of the model

potential energy consists of the sum of the Hartree, image and exchange-correlation terms [6]. The two solutions are matched at the turning point and an eigenvalue is indicated by the continuity of the logarithmic derivative at the matching point, i.e. when the matching tolerance $f=\left[\psi_{n}^{<}\left(z_{m}-h\right)-\psi_{n}^{>}\left(z_{m}+h\right)\right] / \psi_{n}^{<}\left(z_{m}\right)$, where $h$ is the mesh size in the confining direction, is less than $5 \times 10^{-5}$. A simple eigenvalue search is used until $f$ is a monotonically decreasing or increasing function and a bisection method is employed within the energy interval that contains an eigenvalue.

The wavevector-dependent matrix elements are next found for Coulomb scattering from depletion layer and/or oxide/interface charges, surface-roughness scattering and scattering from the various modes of lattice vibrations (acoustic and zero- and first-order nonpolar optical phonons) $[4,7]$. Screened matrix elements for Coulomb and surface-roughness scattering are calculated using the Random Phase Approximation (RPA). Due to the loss of translational invariance in the confining direction, the dielectric function for a Q2D system becomes a fourth rank tensor which requires inversion of a $\mathrm{N} \times \mathrm{N}$ matrix. For (100) sili- con, the size of this matrix is precisely $\mathrm{N}=n_{1}{ }^{2}+\mathrm{n}_{2}{ }^{2}$ where $n_{1}\left(n_{2}\right)$ is the number of subbands from the first (second) set of equivalent valleys with heavy (light) mass perpendicular to the interface. We first evaluate the diagonal (intrasubband) terms of the screened scattering potentials from the bare ones by inverting the linear problem

$$
\begin{aligned}
W_{i i}^{\text {eff }}(\mathbf{q}, \omega) & =V_{i i}^{\text {bare }}(\mathbf{q}) \\
& +\frac{1}{q} \sum_{n} F_{i i, n n}(\mathbf{q}) q_{n n}^{s}(\mathbf{q}, \omega) W_{n n}^{e f f}(\mathbf{q}, \omega),
\end{aligned}
$$

where $F_{i j, n m}(\mathbf{q})$ are the form-factors due to the finite extent of the electron gas in the confining direction and $q_{n n}^{s}(\mathbf{q}, \omega)$ are the screening wavevectors [4]. The off-diagonal (intersubband) terms are then obtained from

$$
\begin{aligned}
W_{i j}^{e f f}(\mathbf{q}, \omega)= & V_{i j}^{\text {bare }}(\mathbf{q})+ \\
& \frac{1}{q} \sum_{n} F_{i j, n n}(\mathbf{q}) q_{n n}^{s}(\mathbf{q}, \omega) W_{n n}^{e f f}(\mathbf{q}, \omega) .
\end{aligned}
$$

Since the matrix elements for Coulomb scattering decrease rapidly with the wavevector, a nonuniform mesh in momentum space is generated with the coordi- 
nate transformation $q_{\text {new }}=q_{\text {old }}^{2} /\left(q_{\text {old }}+a\right)$, where $q_{\text {old }}$ is a wavevector on the uniform mesh, $q_{\text {new }}$ is the corresponding one on the nonuniform mesh and $a=2 \times$ $10^{9} \mathrm{~m}^{-1}$ is an adjustable parameter. In the second step, we evaluate frequency and kinetic energy dependent scattering-induced broadening of the states [4]

$$
\begin{aligned}
\Gamma_{n}\left(\varepsilon_{\mathbf{k}}, \omega\right)=\sum_{m} \frac{m^{*}}{4 \pi^{2} \hbar^{2}} \int_{0}^{\infty} d \varepsilon_{\mathbf{q}} \\
\frac{\Gamma_{m}\left(\varepsilon_{\mathbf{q}}, \omega\right)}{\left[\hbar \omega-\varepsilon_{\mathbf{q}}-\varepsilon_{m}\right]^{2}+\Gamma_{m}^{2}\left(\varepsilon_{\mathbf{q}}, \omega\right)} \int_{0}^{2 \pi} d \varphi T_{n m}\left(\varepsilon_{\mathbf{k}-\mathbf{q}}\right),
\end{aligned}
$$

where $\mathrm{T}_{n m}\left(\varepsilon_{\mathbf{k - q}}\right)$ is the sum of the squared matrix elements of all scattering processes. The first Born approximation results are used as an initial guess in this iterative procedure. Once the system of integral equations (2) is solved, we proceed with the calculation of the density of states (DOS) functions. If selfconsistency is not achieved, we proceed with the solution of the Poisson equation for which we use the approach given in [6] to find the improved Hartree potential. The potential energy profile for the next iteration is obtained by using a fixed-convergence factor scheme for the first two iterations and an extrapolated convergence-factor scheme thereafter. The error criterion for the convergence of the selfconsistent field iterations is that the absolute value of the difference between the input and output potentials at each grid point is less than $0.01 \mathrm{meV}$.

After achieving self-consistency, we iteratively solve the system of Fredholm integral equations (that result from the full Bethe-Salpeter equation)

$$
\begin{aligned}
& \Lambda_{n}\left(\varepsilon_{\mathbf{k}}, \omega\right)=1+\sum_{m} \sum_{\mathbf{q}} \frac{\mathbf{k} \cdot \mathbf{q}}{k^{2}} T_{n m}(\mathbf{k}-\mathbf{q}) \\
& \frac{a_{m}\left(\varepsilon_{\mathbf{q}}, \omega\right)}{2 \Gamma_{m}\left(\varepsilon_{\mathbf{q}}, \omega\right)} \Lambda_{m}\left(\varepsilon_{\mathbf{q}}, \omega\right)
\end{aligned}
$$

for the kernel functions $\Lambda_{n}\left(\varepsilon_{\mathbf{k}}, \omega\right)$ which appear in the expression for the dc-conductivity

$$
\begin{aligned}
\sigma_{2 \mathbf{D}}=2 \frac{e^{2}}{h} \sum_{n} \int d & \left(-\frac{\partial n_{F}}{\partial \omega}\right) \\
& \int_{0}^{\infty} \frac{d \varepsilon_{\mathbf{k}}}{2 \pi} \varepsilon_{\mathbf{k}} \Lambda_{n}\left(\varepsilon_{\mathbf{k}}, \omega\right) \frac{a_{n}\left(\varepsilon_{\mathbf{k}}, \omega\right)}{2 \Gamma_{n}\left(\varepsilon_{\mathbf{k}}, \omega\right)},
\end{aligned}
$$

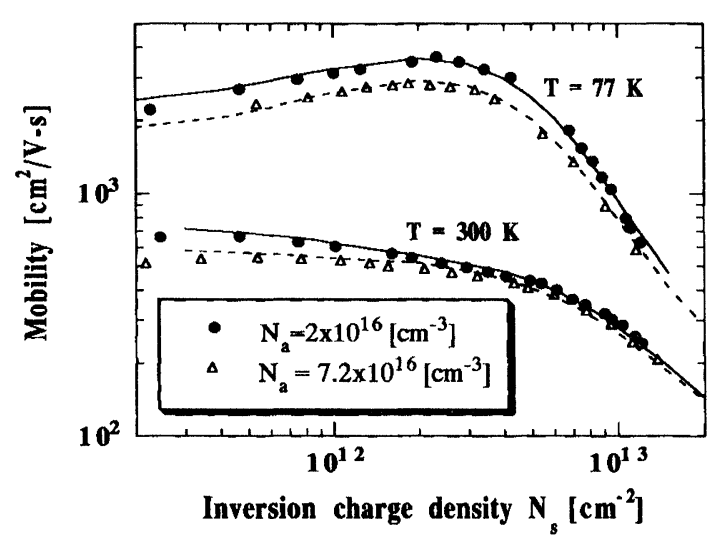

FIGURE 2 Mobility versus inversion charge density at $T=77 \mathrm{~K}$ and $\mathrm{T}=300 \mathrm{~K}$

where $n_{F}(\omega)$ is the Fermi-Dirac distribution function. The average or effective mobility is then calculated from $\mu_{e f f}=\sigma_{2 \mathrm{D}} / e N_{s}$, where $N_{s}$ is the total sheetcharge density.

The mobility is shown in Fig. 2 and compared with experimental data from $n$-channel MOSFET's fabricated on (100) Si wafers. The filled circles and open triangles represent the experimental mobilities for devices with $N_{a}=2 \times 10^{16} \mathrm{~cm}^{-3}$ and $N_{a}=7.2 \times 10^{16}$ $\mathrm{cm}^{-3}$, respectively [8]. The solid and dashed lines are the corresponding simulation results. For both devices, the interface-trap density is estimated to be $N_{i t}=1.75 \times 10^{11} \mathrm{~cm}^{-2}$. Coupling constants for deformation potential and non-polar optical phonon scattering (zero- and first-order interactions) and the corresponding phonon energies, used in the calculations, are given in Refs. [3,9]. Very good agreement between the experimental data and the simulation results throughout the whole inversion charge density region can be observed. The small discrepancy at low values of $N_{s}\left(N_{s}<5 \times 10^{11} \mathrm{~cm}^{-2}\right)$ might be due to the following two reasons: (1) the application of finite drain voltage leads to a decrease in the carrier concentration near the drain causing a lower value for the experimental mobility; (2) a three subband approximation $\left(n_{1}=2\right.$ and $\left.n_{2}=1\right)$, used at present, overestimates the mobility in the low inversion-charge density region where a small fraction of the inversion layer electrons actually resides in the higher-lying subbands. 
Present simulation results suggest that, even at room temperature, Coulomb scattering from interface-trap and depletion layer charges degrades the mobility at low surface fields. The proper treatment of the anisotropy of the deformation potential interaction at $\mathrm{T}=300 \mathrm{~K}$ leads to mobilities that are in close agreement with the experimental data in the range of low-to-medium densities $\left(\approx 10^{11}-10^{12} \mathrm{~cm}^{-2}\right)$, where acoustic phonon scattering limits the mobility. We also find that the importance of surface-roughness scattering shifts toward higher surface fields with increasing temperature. In order to explain the experimental data of Takagi et al. [8] at high surface fields, temperature-dependent roughness parameters have been adopted. In other words, good agreement with the experimental data is achieved by using an exponential model for the autocovariance function with $\Delta=0.3 \mathrm{~nm} \quad(\Delta=0.25 \mathrm{~nm})$ and $\zeta=1.5 \mathrm{~nm}$ $(\zeta=1.4 \mathrm{~nm})$ at $\mathrm{T}=300 \mathrm{~K}(\mathrm{~T}=77 \mathrm{~K})$, as well as the Ando model [6] for the surface-roughness matrix element. These roughness dimensions are comparable to the TEM results obtained by Goodnick et al. [10].

The shape of the cumulative DOS function for the device with $N_{a}=2 \times 10^{16} \mathrm{~cm}^{-3}$ is shown in Fig. 3 . For low values of $N_{s}\left(=N_{s 1}\right)$, strong Coulomb scattering and small subband separation eliminates plateau regions in the DOS curve. The steeper rise of the DOS curve near subband thresholds for $N_{s}=N_{s 2}$ is a conse-

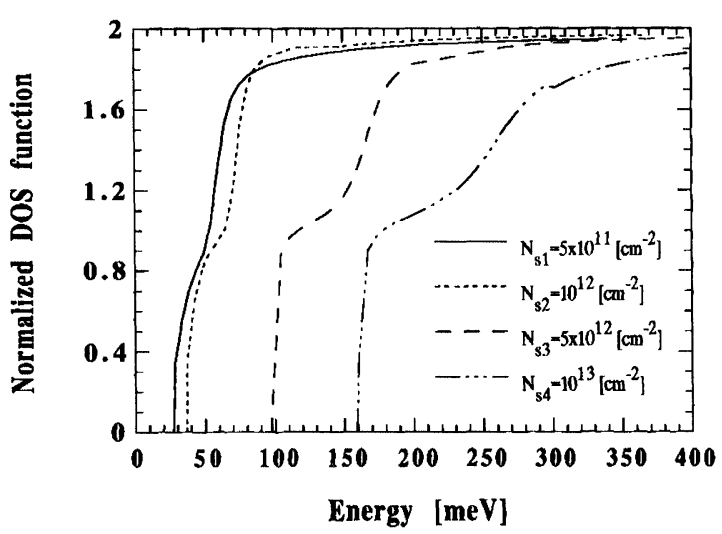

FIGURE 3 Cumulative DOS function for the lowest two subbands of the two equivalent valleys with heavy mass perpendicular to the interface versus energy at $\mathrm{T}=300 \mathrm{~K}$ quence of the decreased importance of Coulomb scattering. At higher values of $N_{s}\left(=N_{s 3}\right)$, the increased subband separation and reduced intersubband scattering allows the plateau regions in the DOS curves to be observed.

In conclusion, simulation results for the mobility -suggest that the theoretical model correctly predicts both the order of magnitude and the overall mobility behavior at various temperatures. We also find that for energies near the subband thresholds, the broadening of the states is comparable to the kinetic energy of the carriers. This leads to modification of the DOS function from its ideal step-like behavior and influences the population of various subbands which, in turn, modifies the mobility.

\section{References}

[1] S. C. Sun, and J. D. Plummer, "Electron Mobility in Inversion and Accumulation Layers on Thermally Oxidized Silicon Surfaces," IEEE Trans. Electron Dev. ED-27, August 1980.

[2] J. A. Cooper, Jr., and D. F. Nelson, "High-Field Drift Velocity of Electrons at the $\mathrm{Si}-\mathrm{SiO}_{2}$ Interface as Determined by a Time-of-Flight Technique," J. Appl. Phys. 54, March 1983.

[3] M. V. Fischetti, and S. E. Laux, "Monte Carlo Study of Electron Transport in Silicon Inversion Layers," Phys. Rev. B 48, July 1993.

[4] D. Vasileska-Kafedziska, PhD Thesis, Arizona State University, December 1995.

[5] S. E. Koonin, and D. C. Meredith, Computational Phys ics, New York: Addison-Wesley Publishing Company, Inc., Fourth Ed. 1993.

[6] T. Ando, A. B. Fowler, and F. Stern, "Electronic Properties of Two-Dimensional Systems," Rev. Mod. Phys. 54, April 1982.

[7] D. K. Ferry, Semiconductors, New York: Macmillan Publishing Company, First Ed. 1991.

[8] S. Takagi, A. Toriumi, M. Iwase, and H. Tango, "On the Universality of Inversion Layer Mobility in Si MOSFET's," IEEE Trans. Electron Dev. 41, December 1994.

[9] D. K. Ferry, "Hot-Electron Effects in Silicon Quantized Inversion Layers," Phys. Rev. B 14, December 1976.

[10] S. M. Goodnick, D. K. Ferry, C. W. Wilmsen, Z. Liliental, D. Fathy and O. L. Krivanek, "Surface Roughness at the $\mathrm{Si}(100)-\mathrm{SiO}_{2}$ Interface," Phys. Rev. B 32, December 1985.

\section{Biographies}

Dragica Vasileska is Assistant Professor in the Department of Electrical Engineering at Arizona State University. Her research interests include semiconductor device physics, device simulation, semiconductor transport theory and quantum transport in quasi-two-dimensional structures. She is currently working on transport through quantum dot structures. 
She is a member of the Phi Kappa Phi honor society and American Physical Society.

Terry Eldridge is a graduate student in the Electrical Engineering Department at Arizona State University, and is employed full-time with McDonnell Douglas Helicopter Systems. His research and engineering interests have included experimental high energy physics, real-time software design and development, radar and signal processing, radio communications, on-aircraft airspeed sensors, and semiconductor transport theory. He is a member of the American Physical Society and the Institute of Electrical and Electronics Engineering.

Paolo Bordone is a faculty research associate in the Dipartimento di Fisica ed Instituto Nazionale di Fisica della Materia, Università di Modena, Italy. His research interests include charge transport in semiconductors, phonon population fluctuations in polar semiconductors and their effects on charge transport phenomena, collision duration times for polar optical and intervalley scattering, etc. He is a member of the American Physical Society.

David K. Ferry is Regent's Professor of Engineering at Arizona State University. His research encompasses transport physics and modeling of submicron devices, particularly the inclusion of quantum effects, and electron-beam lithography of ultra-submicron devices, where his group has fabricated HEMT's with gate lengths as short as $25 \mathrm{~nm}$. He is a fellow of both the American Physical Society and the Institute of Electrical and Electronics Engineers. 

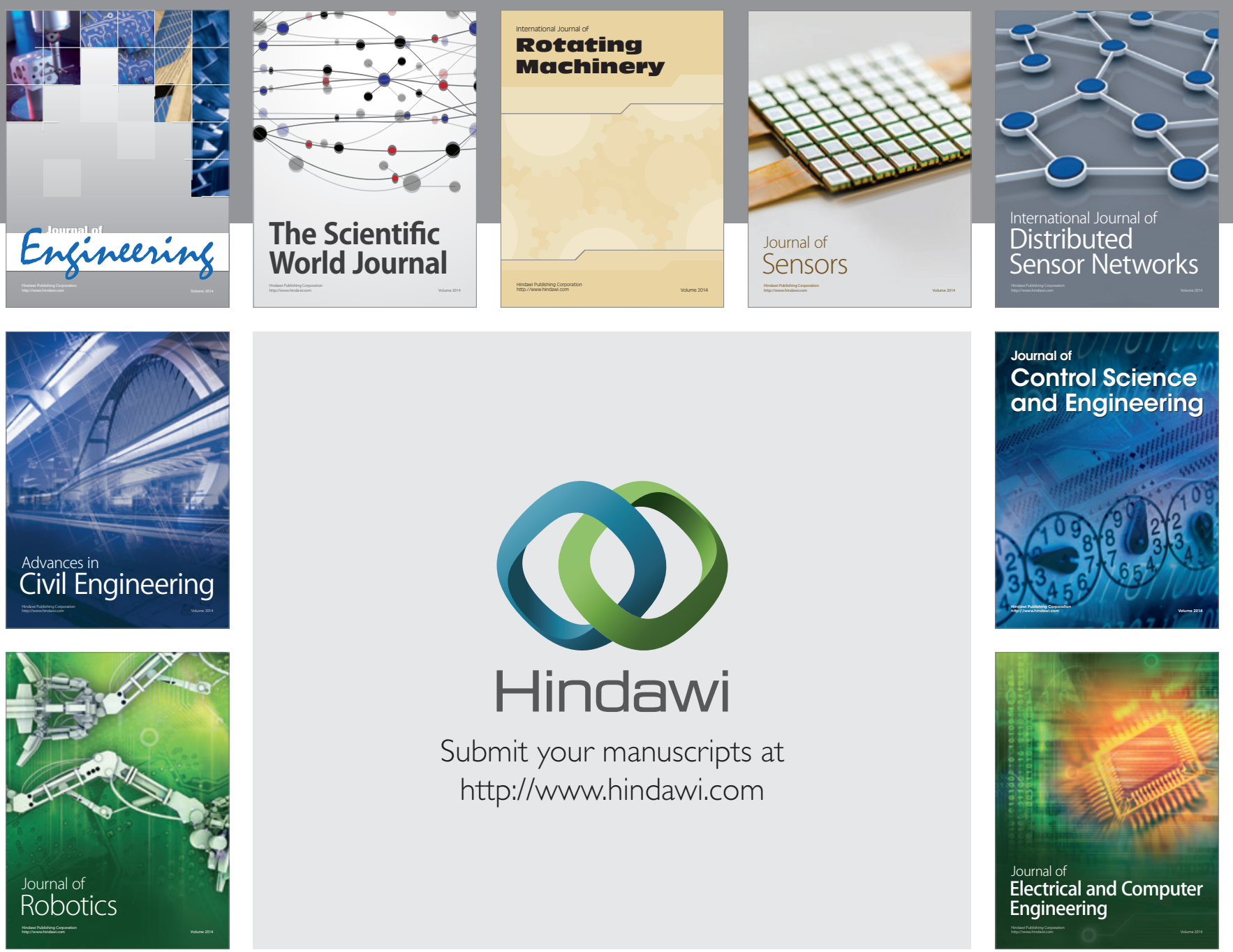

Submit your manuscripts at

http://www.hindawi.com
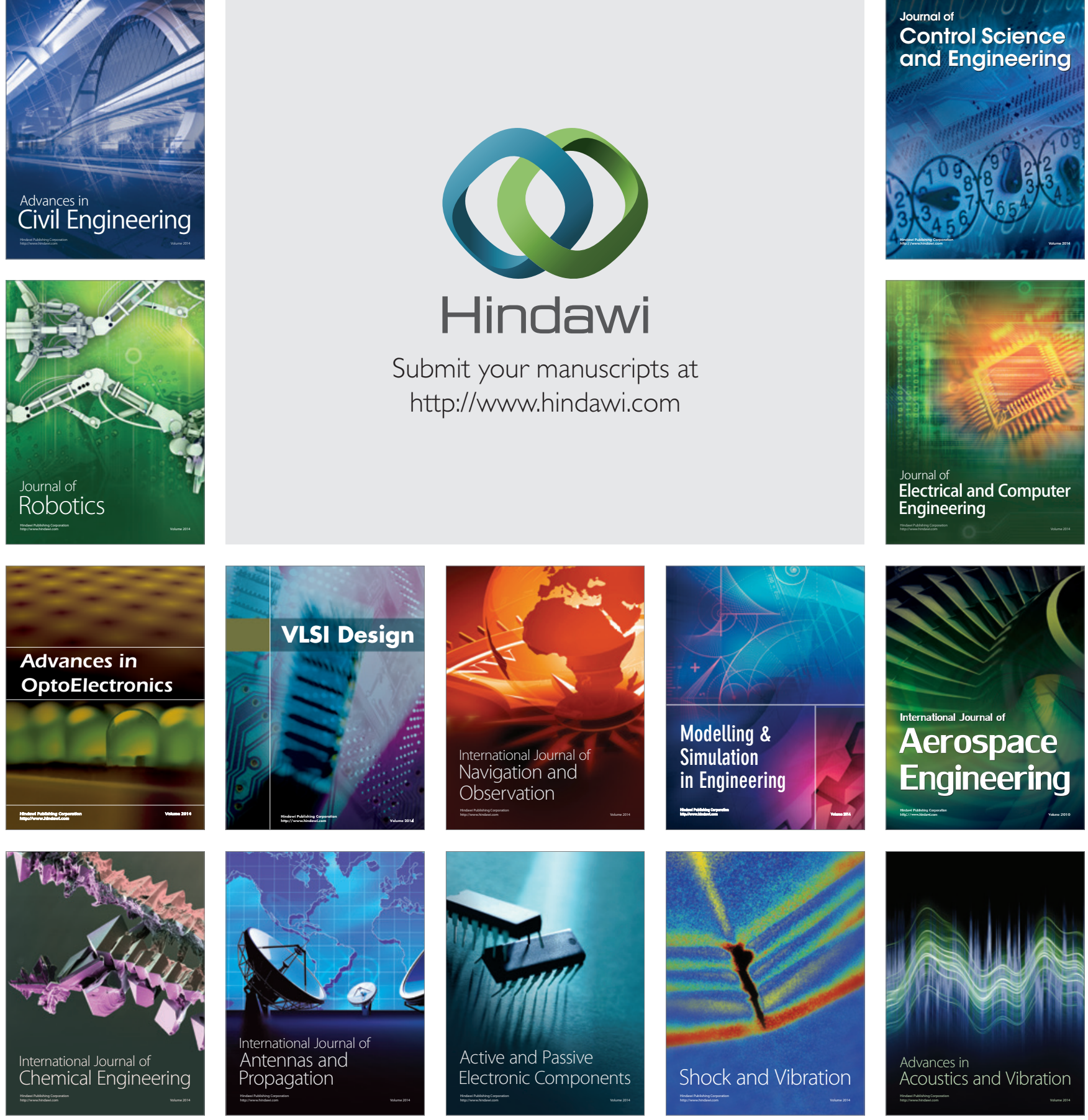